\title{
Analysis and regulation of the stress-strain state of structures with reliability
}

\author{
Yurii Tryapitsin ${ }^{1, *}$, Viktor Pakhomov ${ }^{1}$, Sergei Voinov ${ }^{1}$ \\ ${ }^{1}$ Far Eastern State Transport University, Serysheva st., 47, Khabarovsk, 680021, Russia
}

\begin{abstract}
It is offered an algorithm for analysis and regulation of the stress-strain state of building constructions and soils of the foundations of structures at designing, building and exploitation, at setting of the mode of exploitation for providing of the required level of reliability, when tensions in them differ from calculation values. Possible options for secured or unsecured reliability are considered. The algorithm consists in determining the necessary decrease in the design stresses or increase the bearing capacity of structure. Offered algorithm is an estimation of necessary displacement of density of distribution of current tension or density of distribution of bearing strength for the achievement of the required level of reliability of the constructions and soil of base of the structure. Further research will be devoted to the consideration of case with unsecured reliability, for which it is necessary to assess the necessary displacement of the distribution density of the existing stresses or the distribution density of the bearing capacity to achieve the required level of reliability of the building constructions and soil of base of the structure.
\end{abstract}

\section{Introduction}

At designing, building and exploitation of different engineering building the normative levels of reliability are set $[H]$, the indicated objects must correspond that (Russian Standard: GOST R 53778-2010, GOST R 54257-2010, ISO 2394:2015). There are many works devoted to assessing the reliability of various structures, taking into account various factors [1-22], as well as optimization problems of reliability [23-25]. Thus the estimation of bearing strength of constructions and grounds of building is performed on the Building rules by the method of the marginal states in the determined raising, and the algorithm of analysis of their stress-strain state and his adjusting is absent from positions of providing of the required reliability $[H]$. At the estimation of reliability of actual stress-strain state $H$ the cases of $H$ are possible $H>[H]$ or $H<[H]$ at satisfaction or dissatisfaction of requirements on the first and second group of the marginal states in the determined raising, regulated in Building rules and other normative documents.

The problem to work out the algorithm for analysis and regulation of the stress-strain state of building constructions and soils of the foundations of structures from positions of providing of the required reliability is set.

\footnotetext{
* Corresponding author: TryapitsinYV@mail.ru
} 


\section{Materials and Methods}

In basis of calculations on durability the method of the marginal states, in that for the estimation of capacity of building constructions and soil of the base a parameter is used, being a difference between bearing strength of element (founding) of $\mathrm{R}$ and current tension in it $\sigma$, and named the function of tension (by reserve of durability), is fixed in the probabilistic raising:

$$
\psi=R-\sigma
$$

It is accepted within the framework of probabilistic approach, that descriptions of current tension and bearing strength of building constructions and soil of the foundations of structures carry casual character and inferior to the normal law of distribution. Descriptions of distribution of these sizes are the expected value $\bar{\sigma}, \bar{R}$ and standard deviation $S_{\sigma}, S_{R}$. The next concepts of probabilistic approach of calculations are used on durability.

Reliability is probability of faultless work of element or building on the whole during the set term of exploitation (tenure of employment). A refuse is a random event consisting in violation of capacity of object.

Probability of refuse is a difference between unit, qualitatively characterizing the capacity of object and by its reliability:

$$
P=1-H \text {. }
$$

A quantile of reliability (refuse) is a value of casual size: current tension $\sigma_{p}$, bearing strength $R_{p}$ or reserve of durability $\psi_{p}=R_{p}-\sigma_{p}$ (Fig.1.A) corresponding to the set value of function of distribution of this size (i.e. to the set level of reliability $H$ or set probability of refuse $P=1-H$ ). The index of " $P$ " in denotations $\sigma_{p}$ and $R_{p}$ specifies on the level of reliability, with that these sizes are determined. Quantile of standard normal distribution, $t_{p}$, is a parameter of function $\Phi$ of distribution of the casual size (current tension $\sigma$, of bearing strength $R$, or function of tensions $\psi=R-\sigma$ ) rationed as follows (Fig.1.B):

$$
t_{\mathrm{p}}=\frac{\sigma_{p}-\bar{\sigma}}{S_{\sigma}}, t_{\mathrm{p}}=\frac{R_{p}-\bar{R}}{S_{R}}, t_{\mathrm{p}}=\frac{\psi_{p}-\bar{\psi}}{S_{\psi}}
$$

Thus

$$
\bar{t}=0, S_{\sigma}=S_{R}=S_{\psi}=1 \text {. }
$$

The function of standard normal distribution possesses next property: $\Phi\left(-t_{p}\right)=1-\Phi\left(t_{p}\right)$, i.e. for the same level of reliability and refuse their quantiles are equal on a size and opposite on a sign. Knowing the quantile of refuse it is possible to find the level of reliability and vice versa.

At the estimation of probability of refuse, when $\psi=0$, quantile of probability of refuse:

$$
t_{\mathrm{p}}=\frac{0-\bar{\psi}}{S_{\psi}}=\frac{-(\bar{R}-\bar{\sigma})}{\sqrt{S_{R}^{2}+S_{\sigma}^{2}}},
$$

And corresponding to it quantile of function of reliability:

$$
t_{p}^{\prime}=-t_{p}=-\frac{0-\bar{\psi}}{S_{\psi}}=-\frac{-(\bar{R}-\bar{\sigma})}{\sqrt{S_{R}^{2}+S_{\sigma}^{2}}}=\frac{\bar{R}-\bar{\sigma}}{\sqrt{S_{R}^{2}+S_{\sigma}^{2}}} .
$$

Quantile of the rationed casual size, $t_{p}{ }^{\prime}$, determines the number of standards, on that the casual size of current tension $\sigma$ or bearing strength $R$, or function of tensions $\psi=R-\sigma$, from the mean value of distribution $\sigma, R$ or $\psi$, to get the calculation value of current tension $\sigma_{p}$, bearing strength $R_{p}$ or reserve of durability $\psi_{p}=R_{p}-\sigma_{p}$ corresponding to the et (required) 
level reliability $[H]$ or to the set probability of refuse $[P]=1-[H]$ (Fig.2). Sometimes $t_{p}{ }^{\prime}$ is named safety feature.
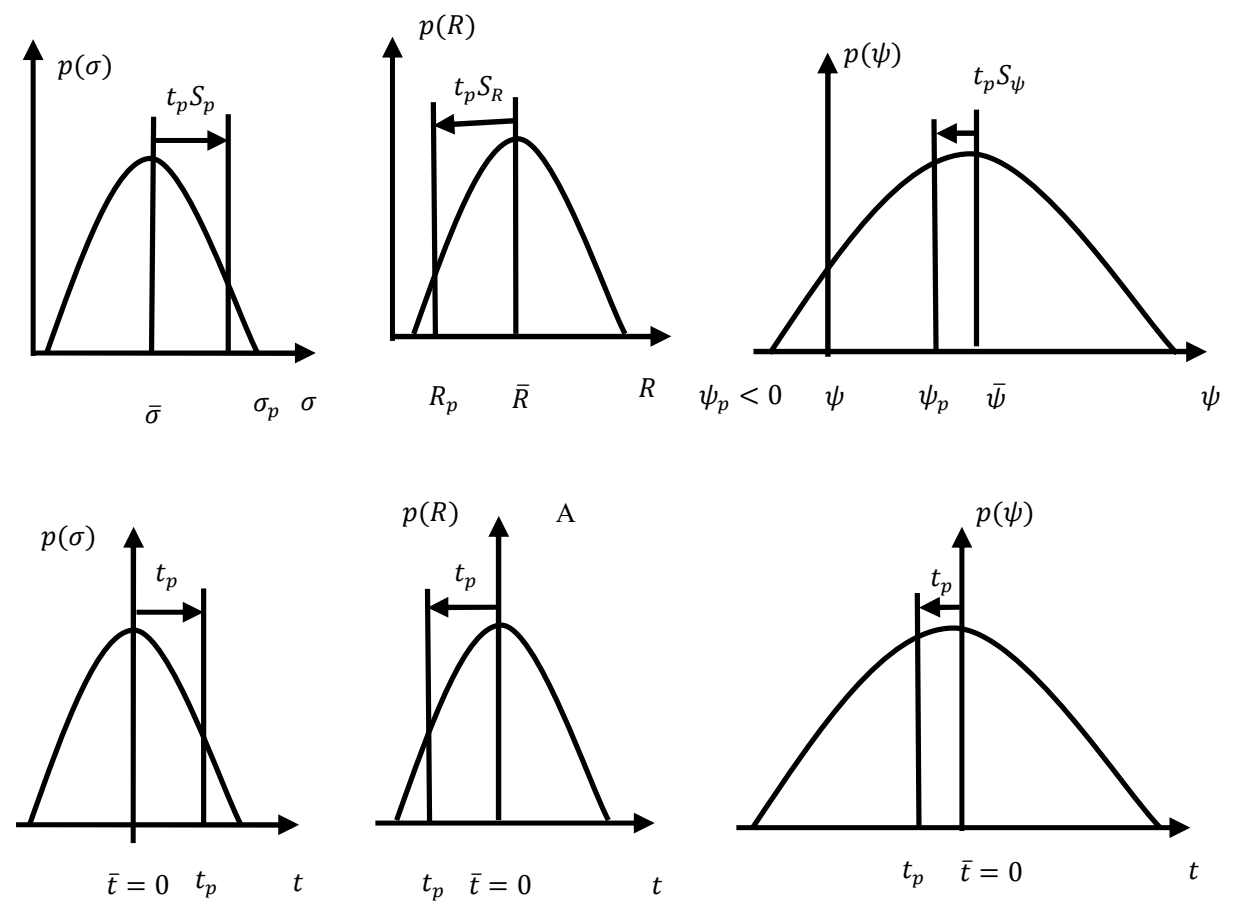

B

Fig.1. Closeness of probability of distribution of current tension of $p(\sigma)$, bearing strength of $p(R)$, or functions of tensions of $p(\psi=R-\sigma)$ : A) - initial sizes, $\mathrm{B})$ - the rationed sizes

Next calculation cases (Fig.2) are possible:

A) $\sigma_{p}<R_{p}$ at any level of reliability of $H$ and refuse of $P$;

B) $\sigma_{p}>R_{p}$ at some level of $H$ and $P, \psi_{p}=R_{p}-\sigma_{p}<0$, that is not provided for this technical state of object (correlations of current tension and bearing strength), at this level of $H$ durability is not provided;

C) $\sigma_{p}<R_{p}$ at some level of reliability of $H$ and refuse of $P, \psi_{p}=R_{p}-\sigma_{p}>0$, that can be $H<>[H], P><[P]$, look the case of D);

D) $\sigma_{p}<R_{p}$ at some levels of reliability of $H_{l}$ and $H_{2}$, thus $H_{l}<H_{2}$, accordingly: $t_{p l}<t_{p 2}$. If $H_{2}=[H]$, that at $H_{l}<[H]$, reliability of object (with $H_{l}$ ) is not provided, in spite of the fact that $\psi_{p}=R_{p}-\sigma_{p}>0$. If $H_{1}=[H]$, that at $H_{2}>[H]$, reliability of object (with $H_{2}$ ) is provided.

An area of crossing of charts of $p\left(\sigma_{p}\right)$ and $p\left(R_{p}\right)$ is an area of probability of refuse.

Calculation tensions and calculation resistances, corresponding to the required level of reliability, are determined on formula

$$
\begin{aligned}
& \sigma_{p}=\bar{\sigma}+t_{p}^{\prime} S_{\sigma} \\
& R_{p}=\bar{R}-t_{p}^{\prime} S_{R}
\end{aligned}
$$

Descriptions of distribution of current tension, $\bar{\sigma}, \mathrm{S}_{\sigma}$, and resistances, $\bar{R}, \mathrm{~S}_{\mathrm{R}}$, determined from formulas:

$$
\sigma_{n}=\bar{\sigma}+\gamma_{n} S_{\sigma}
$$




$$
\begin{aligned}
& \sigma_{p}=\bar{\sigma}+\gamma_{\mathrm{p}} S_{\sigma} \\
& R_{y n}=\bar{R}-\gamma_{n} S_{R} \\
& R_{y}=\bar{R}-\gamma_{\mathrm{p}} S_{R}
\end{aligned}
$$

where $\gamma_{p}=3,09$ and $\gamma_{n}=1,65$ are quantiles of calculation $(99,9 \%)$ and normative $(95 \%)$ material security. $\sigma_{p}, R_{y}$ and $\sigma_{n}, R_{y n}$ - norms are calculation and normative tensions and resistances of the same securities. For other values of normative securities of the separate external loading $\sigma_{n}$ it is possible to count in $\sigma_{n}$ for $H=0,95$.

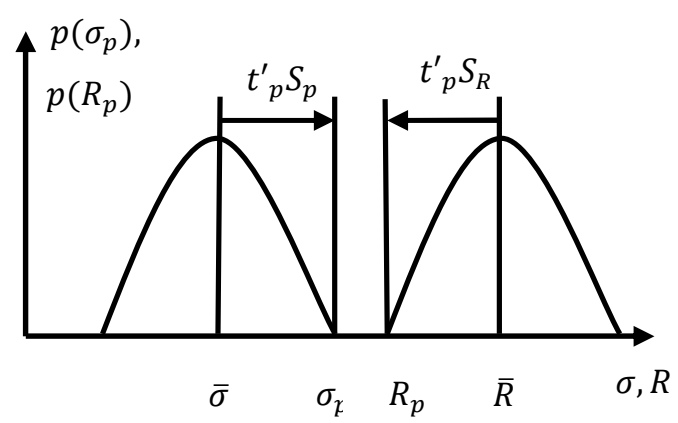

A

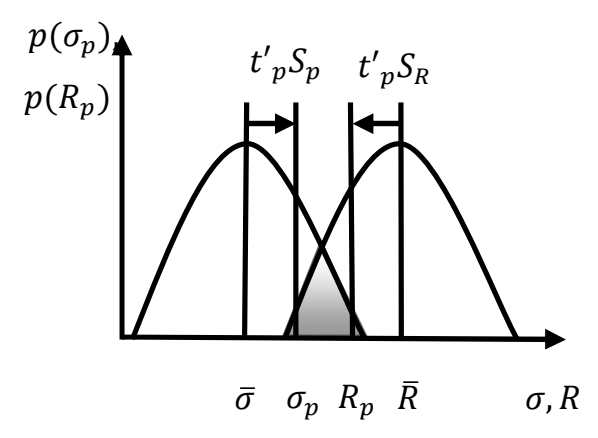

$\mathrm{C}$

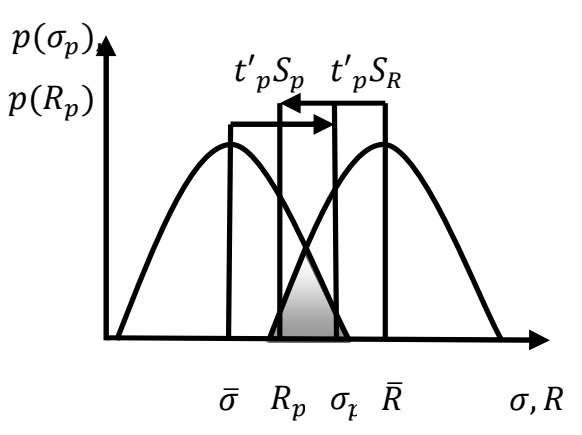

B

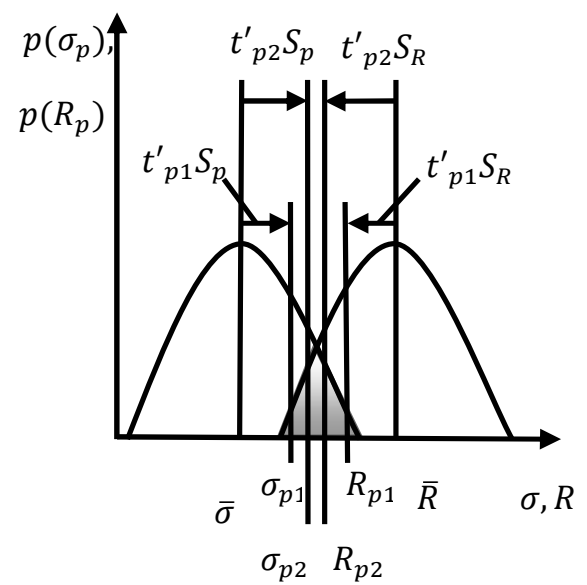

$\mathrm{D}$

Fig.2. Calculation cases for the estimation of durability in the probabilistic raising

Condition of durability in the probabilistic raising:

In terms of function of tensions (reserve of durability):

$$
\psi_{p}=R_{p}-\sigma_{p}>0
$$

In terms of probability of refuse:

$$
P=P(\psi \leq 0)=P(R-\sigma \leq 0)=\Phi\left(t_{\mathrm{p}}=\frac{0-\bar{\psi}}{S_{\psi}}=\frac{-(\bar{R}-\bar{\sigma})}{\sqrt{S_{R}^{2}+S_{\sigma}^{2}}}\right) \leq[P]
$$

In terms of level of reliability: 


$$
H=P(\psi>0)=P(R-\sigma>0)=\Phi\left(t_{p}^{\prime}=-t_{p}=\frac{\bar{R}-\bar{\sigma}}{\sqrt{S_{R}^{2}+S_{\sigma}^{2}}}\right) \geq[H]
$$

Task. It is need to estimate reliability of soil of founding of building.

Soil of founding: loam, calculation resistance $R_{y}=250 \mathrm{kPa}$.

Coefficient of reliability on soil $\gamma_{m}=1.15$.

Normative resistance $R_{y n}=\gamma_{m} R_{y}=1.15-250=287.5 \mathrm{kPa}$.

Average value of current stresses for pressure sensors in the ground $\bar{\sigma}=200 \mathrm{kPa}$. Normative tensions

$$
\sigma_{n}=\bar{\sigma} \cdot \frac{\gamma_{\mathrm{p}}-\gamma_{\mathrm{H}}}{\gamma_{\mathrm{p}}-\gamma_{f} \cdot \gamma_{n}}=200 \cdot \frac{(3.09-1.65)}{(3.09-1.1 \cdot 1.65)}=225.88 \mathrm{kPa} .
$$

where $\gamma_{p}=3.09$ and $\gamma_{n}=1.65$ are quantiles of calculation $(99.9 \%)$ and normative $(95 \%)$ material well-being.

$\gamma_{f}=1,1$ is a coefficient of reliability on loading.

Calculation tensions

$$
\sigma_{\mathrm{p}}=\gamma_{f} \sigma_{n}=1.1 \cdot 225,88=248.47 \mathrm{kPa}
$$

Standard deviation (standard) of current tension:

$$
S_{\sigma}=\sigma_{n} \cdot \frac{\left(\gamma_{f}-1\right)}{\left(\gamma_{\mathrm{p}}-\gamma_{n}\right)}=225.88 \cdot \frac{(1.1-1)}{(3.09-1.65)}=15.69 \mathrm{kPa} .
$$

Coefficient of variation on tension:

$$
C_{V \sigma}=\frac{S_{\sigma}}{\bar{\sigma}}=\frac{\left(\gamma_{f}-1\right)}{\left(\gamma_{\mathrm{p}}-\gamma_{f} \cdot \gamma_{n}\right)}=\frac{(1.1-1)}{(3.09-1.1 \cdot 1.65)}=0.078
$$

Descriptions of distribution of durability became:

Mean value of durability:

$$
\bar{R}=R_{y n} \cdot \frac{\left(\gamma_{\mathrm{p}}-\frac{\gamma_{n}}{\gamma_{m}}\right)}{\left(\gamma_{\mathrm{p}}-\gamma_{n}\right)}=287.5 \cdot \frac{\left(3.09-\frac{1.65}{1.15}\right)}{(3.09-1.65)}=330.47 \mathrm{kPa} .
$$

Standard deviation (standard) of durability:

$$
S_{R}=R_{y n} \cdot \frac{\left(1-\frac{1}{\gamma_{m}}\right)}{\left(\gamma_{\mathrm{p}}-\gamma_{n}\right)}=287.5 \cdot \frac{\left(1-\frac{1}{1.15}\right)}{(3.09-1.65)}=26.04 \mathrm{kPa}
$$

Coefficient of variation on durability:

$$
C_{V R}=\frac{S_{\sigma}}{\bar{\sigma}}=\frac{\left(1-1 / \gamma_{m}\right)}{\left(\gamma_{\mathrm{p}}-\gamma_{n} / \gamma_{m}\right)}=\frac{(1-1 / 1.15)}{(3.09-1.65 / 1.15)}=0.079
$$

Coefficient of reliability on purpose:

$$
\gamma_{n}^{1}=1.15
$$

Condition of verification of bearing strength of founding:

$$
\sigma_{\mathrm{p}} \leq \frac{\gamma_{\mathrm{c}}^{1}}{\gamma_{n}^{1}} \cdot R_{y}
$$

where $\gamma_{c}^{1}$ - is a coefficient of terms of work of soil of founding, $\gamma_{c}^{1}=0.9$.

$$
248.47 \mathrm{kPa}>\frac{0.9}{1.15} \cdot 250=195.65 \mathrm{kPa},
$$

Consequently, bearing strength of founding is not provided.

Quantile of function of reliability at the normal law of distribution: 


$$
t_{p}=\frac{\bar{R}-\bar{\sigma}}{\sqrt{S_{R}^{2}+S_{\sigma}^{2}}}=\frac{330.47-200}{\sqrt{26.04^{2}+15.69^{2}}}=4.29
$$

Reliability of element:

$$
\mathrm{H}_{\mathrm{K}}=\Phi\left(t_{p}\right)=\Phi(5,19)=0.999991066
$$

Normative level of building reliability $[H]=0,9999995$.

$H=0,999991066<[H]=0,9999995$, consequently, reliability of element is not provided.

Probability of refuse of element:

$$
\mathrm{P}_{\mathrm{K}}=1-\mathrm{H}_{\mathrm{K}}=1-0.999991066=0.000008934
$$

We have a calculation case of "B" Fig.2 possible at planning.

For providing of the required level of reliability of the examined founding of building either the decline of calculation tensions $\Delta \sigma_{p}$ (due to a unloading or due to the jumboizing of section) or increase of bearing strength $\Delta R_{y}$ (due to the increase of bearing strength of founding) is needed. On some size.

Size $\Delta \sigma_{p}$ or $\Delta R_{y}$ will be determined from next expressions.

$$
\begin{gathered}
t_{p}^{[H]}=\frac{\bar{R}-\bar{\sigma}}{\sqrt{S_{R}^{2}+S_{\sigma}^{2}}}=\Phi^{-1}([\mathrm{H}])=\Phi^{-1}(0.9999995)=4.895 \\
t_{p}^{[H]} \cdot \sqrt{S_{R}^{2}+S_{\sigma}^{2}}=\bar{R}-\bar{\sigma} .
\end{gathered}
$$

Decline of calculation tensions required from where:

$$
\begin{gathered}
\bar{\sigma}^{\prime}=\bar{R}-t_{p}^{[H]} \cdot \sqrt{S_{R}^{2}+S_{\sigma}^{2}}=330.47-4.895 \cdot \sqrt{26.04^{2}+15.69^{2}}=181.65 \mathrm{kPa} . \\
\sigma_{p}^{[H]}=\bar{\sigma}^{\prime}+t_{p} \cdot S_{\sigma}=181.65+3.09 \cdot 15.69=230.13 \mathrm{kPa} . \\
\Delta \sigma_{\mathrm{p}}=\sigma_{p}-\sigma_{p}^{[H]}=248.47-230.13=18.34 \mathrm{kPa} .
\end{gathered}
$$

Or the required increase of bearing strength:

$$
\begin{gathered}
\bar{R}^{\prime}=\bar{\sigma}+t_{p}^{[H]} \cdot \sqrt{S_{R}^{2}+S_{\sigma}^{2}}=200+4.895 \cdot \sqrt{26.04^{2}+15.69^{2}}=348.82 \mathrm{kPa} \\
R_{p}^{[H]}=\bar{R}^{\prime}-t_{p} \cdot S_{R}=348.82-3.09 \cdot 26.04=268.35 \mathrm{kPa} \\
\Delta R_{y}=R_{p}^{[H]}-R_{p}=268.35-250=18.35 \mathrm{kPa} .
\end{gathered}
$$

\section{Results}

Possible options for secured or unsecured reliability are considered.

An algorithm for the analysis of the stress-strain state of soils of the foundations of structures and its regulation from the standpoint of ensuring the required reliability is proposed, which consists in determining the necessary decrease in the design stresses or increase the bearing capacity of the soil of the base.

\section{Discussion}

Based on the proposed algorithm for ensuring the required reliability, design case B is considered (Fig.2) with unsecured reliability.

The solution to the problem is obtained by equating the quantile of the reliability function to the quantile of the required level of reliability. 
The proposed algorithm makes it possible to assess reliability and prescribe measures to ensure the required level of reliability not only of base soils, but also of building structures of a structure.

Further research will be devoted to the consideration of case D (Fig. 2) with unsecured reliability.

\section{Conclusion}

Within the framework of the offered probabilistic approach the task of analysis of stress-strain state of building constructions and soils of the foundations of structures and its regulation from positions of providing of the required reliability [H].

Offered algorithm of analysis of stress-strain state and estimation of the technical state of the building constructions and soil of base of the structure from positions of providing of the required reliability $[H]$ it is an estimation of necessary displacement of density of distribution of current tension or density of distribution of bearing strength for the achievement of the required level of reliability of the constructions and soil of base of the structure $[H]$ at the decision of the tasks indicated higher.

Further research will be devoted to the consideration of case D (Fig.2) with unsecured reliability, for which it is necessary to assess the necessary displacement of the distribution density of the existing stresses or the distribution density of the bearing capacity to achieve the required level of reliability of the building constructions and soil of base of the structure [H].

\section{References}

1. Zh. Yang, J. Ching, Applied Mathematical Modelling, 74, 337-349, (2019)

2. C. Wang, H. Zhang, Q. Li, Reliability Engineering \& System Safety, 181, 38-45, (2019)

3. D. Dias-da-Costa, L.A.C. Neves, S. Gomes, S.A. Hadigheh, P. Fernandes, Engineering Structures, 196, 109297, (2019)

4. H. Gazi, C. Alhan, Engineering Structures, 195, 490-507, (2019)

5. J. Ji, Ch. Zhang, Y. Gao, J. Kodikara, Computers and Geotechnics, 111, 22-29, (2019)

6. A. Vojdani, G. H. Farrahi, Theoretical and Applied Fracture Mechanics, 104, 102333, (to be published)

7. A.A. Roubos, R.D.J.M. Steenbergen, T. Schweckendiek, S.N. Jonkman, Structural Safety, 75, 89-109, (2018).

8. D. De-León, L. Loza, International Journal of Disaster Risk Reduction, 40, 101276. 2019

9. T. Zhou, Y. Peng, J. Li, Mechanical Systems and Signal Processing, 131, 592-616, (2019).

10. M. Ghalehnovi, M. Rashki, A. Ameryan, Applied Mathematical Modelling, 77, 1, 829-847, (to be published)

11. S. Chen, Ch. Hou, H. Zhang, L.-H. Han, Thin-Walled Structures, 143, 106192, (to be published)

12. A. E. Aladejare, Y. Wang, Geoscience Frontiers, 9, 6, 1639-1648, (2018).

13. Y. Yuan, W. Han, G. Li, Q. Xie, Q. Guo, Engineering Structures, 197, 109426, (to be published).

14. J. Li, R. Gao, Probabilistic Engineering Mechanics, 56, 14-26, (2019)

15. V. Ho-Huu, D. Duong-Gia, T. Vo-Duy, T. Le-Duc, T. Nguyen-Thoi. Expert Systems with Applications, 102, 262-272, (2018).

16. M. Nassar, L. Guizani, M.-J. Nollet, A. Tahan, Engineering Structures, 197, 109353, (2019) 
17. M.A. Shayanfar, M.A. Barkhordari, M.A. Roudak, Communications in Nonlinear Science and Numerical Simulation, 60, 184-202, (2018)

18. D.-Q. Li, F.-P. Zhang, Z-J. Cao, X.-S. Tang, S.-K. Au, Engineering Geology, 245, 130-140, (2018)

19. Z. Chen, J. Du, J. Yan, P. Sun, K. Li, Y. Li, Engineering Geology, 105232, (2019)

20. J. Szafran, K. Juszczyk, M. Kamiński, Journal of Constructional Steel Research, 154, 278-292, (2019)

21. K. Kubicka, P. Obara, U. Radoń, W. Szaniec, Archives of Civil and Mechanical Engineering, 19, 2, 417-427, (2019)

22. K. Nasrollahzadeh, R. Aghamohammadi, Engineering Structures, 176, 785-800, (2018)

23. M. Mishra, V. Keshavarzzadeh, A. Noshadravan, Structural Safety, 76, 1-14, (2019)

24. S.H. Ghasemi, A.S. Nowak, Engineering Structures, 152, 226-237, (2017)

25. G. das Neves Carneiro, C. Conceição António, Structural Safety, 79, 54-65, (2019) 